First publ. in: Physical Review B, Vol. 72 (2005), Article 180503

\title{
Superconducting proximity effect in a diffusive ferromagnet with spin-active interfaces
}

\author{
A. Cottet and W. Belzig \\ Department of Physics and Astronomy, University of Basel, Klingelbergstrasse 82, 4056 Basel, Switzerland
}

(Received 27 September 2005; published 21 November 2005)

\begin{abstract}
We reconsider the problem of the superconducting proximity effect in a diffusive ferromagnet bounded by tunneling interfaces, using spin-dependent boundary conditions. This introduces for each interface a phaseshifting conductance $G_{\phi}$ which results from the spin dependence of the phase shifts acquired by electrons upon scattering on the interface. We show that $G_{\phi}$ strongly affects the density of states and supercurrents predicted for superconducting/ferromagnetic hybrid circuits. We show the relevance of this effect by identifying clear signatures of $G_{\phi}$ in the data of T. Kontos et al. [Phys. Rev. Lett. 86, 304 (2001), 89, 137007 (2002)].
\end{abstract}

PACS number(s): 74.50.+r, 74.20. $-\mathrm{z}, 73.23 .-\mathrm{b}$

Superconducting/ferromagnetic $(S / F)$ hybrid structures raise the fundamental question of what happens when two phases with different broken symmetries interact. When a $F$ metal with uniform magnetization is connected to a BCS superconductor, the singlet electronic correlations characteristic of the $S$ phase propagate into $F$ via Andreev reflections which couple electrons and holes with opposite spins and excitation energies. In the diffusive case, this propagation occurs on a scale limited by the ferromagnetic exchange field. The decay of the correlations in $F$ is accompanied by oscillations of the superconducting order parameter because the exchange field induces an energy shift between the correlated electrons and holes. ${ }^{1,2}$ This has been observed experimentally through oscillations of the density of states (DOS) in $F,{ }^{3}$ or of the critical current $I_{0}$ through $S / F / S$ structures, ${ }^{4-7}$ with the thickness of $F$ or the temperature. Remarkably, the oscillations of $I_{0}$ have allowed one to obtain $\pi$ junctions, i.e., Josephson junctions with $I_{0}<0,{ }^{8}$ which could find applications in the field of superconducting circuits. ${ }^{9}$

The interface between a ferromagnet and a nonmagnetic material can scatter electrons with spin that is parallel or antiparallel to the magnetization of the ferromagnet with different phase shifts. The spin dependence of the interfacial phase shifts (SDIPS) is a general concept in the field of spindependent transport. The SDIPS implies that spins noncollinear to the magnetization precess during the scattering by the interface. This so-called spin mixing is expected to drastically affect the behavior of $F /$ normal metal systems ${ }^{10}$ when several $F$ electrodes with noncollinear magnetization are used. The same phenomenon is predicted to occur in $F /$ coulomb blockade island, ${ }^{11}$ and $F /$ Luttinger liquid ${ }^{12}$ hybrid circuits. In $S / F$ hybrid systems, ${ }^{13-15}$ the SDIPS is even predicted to affect the system in collinear configurations, due to the coupling of electrons and holes with opposite spins by the Andreev reflections. However, few experimental signatures of the SDIPS have been identified up to now (e.g., Ref. 13 proposes for the data of Ref. 16 an interpretation based on the SDIPS).

In this Rapid Communication, we reconsider the problem of the superconducting proximity effect in a diffusive $F$. Up to now the tunnel $S / F$ contacts used to produce this effect were described (see, e.g., Ref. 2) with spin-independent boundary conditions (BC) derived in Ref. 17 for the spin- degenerate case. Instead of that, we use spin-dependent $\mathrm{BC}$ based on Ref. 15. These BC introduce a phase-shifting conductance $G_{\phi}$ which takes into account the SDIPS. We show that $G_{\phi}$ strongly affects the phase and the amplitude of the oscillations of the DOS or $I_{0}$ with the thickness of $F$. Our approach thus provides a framework for future work on $S / F$ diffusive circuits with tunneling interfaces. We show its relevance by a comparison with the data of Refs. 3 and 5 which shows that strong experimental manifestations of the SDIPS have already been observed through the superconducting proximity effect.

We consider a $S / F$ hybrid circuit with a single $F$ electrode homogeneously magnetized in direction $\vec{z}$. In the diffusive limit, the electrons in a superconducting or ferromagnetic electrode $\alpha$ can be described with quasiclassical and diffusive Green's functions $\check{G}_{\alpha}$ in the Keldysh $\otimes$ Nambu $\otimes$ spin space (we use the notations of Ref. 15). The BC at a $S / F$ interface can be calculated by assuming that the interface potential locally dominates the Hamiltonian, i.e., at a short distance it causes only ordinary scattering (with no particlehole mixing). We characterize this scattering with transmission and reflection amplitudes $t_{n, \sigma}^{S(F)}$ and $r_{n, \sigma}^{S(F)}$ for electrons coming from the $S(F)$ side in channel $n$ with a spin $\sigma$ parallel $(\sigma=\uparrow)$ or antiparallel $(\sigma=\downarrow)$ to $\vec{z}$. In practice, the planar $S / F$ contacts used to induce the superconducting proximity effect in a diffusive ferromagnet are likely to be in the tunnel limit, ${ }^{18,19}$ due, e.g., to a mismatch of band structure between $S$ and $F$, thus we assume $T_{n}=\Sigma_{\sigma}\left|t_{n, \sigma}^{S}\right|^{2} \ll 1$. We also consider that the system is weakly polarized. Following Ref. 15 and 20 , the $\mathrm{BC}$ at the right-hand side $F$ of a $S / F$ interface is

$$
\begin{aligned}
2 g_{F} \check{G}_{F} \frac{\partial \check{G}_{F}}{\partial x}= & {\left[G_{t} \check{G}_{S}+i G_{\phi} \sigma_{z} \check{\tau}_{3}+\frac{G_{M R}}{2} \check{D}_{+}, \check{G}_{F}\right] } \\
& +\left[i G_{\chi} \check{G}_{S} \check{D}_{-}+i G_{\xi} \check{D}_{-} \check{G}_{F}, \check{G}_{F}\right]
\end{aligned}
$$

with $\check{D}_{ \pm}=\sigma_{z} \check{\tau}_{3} \check{G}_{S} \pm \check{G}_{S} \sigma_{z} \check{\tau}_{3}$. Here, $\sigma_{z}$ and $\check{\tau}_{3}$ are Pauli matrices in spin and Nambu space, respectively. The conductivity of $F$ times the area of the junction, noted $g_{F}$, is assumed to be spin independent. The coefficient $G_{t}=G_{Q} \Sigma_{n} T_{n}$ is the tunneling conductance, $G_{M R}=G_{Q} \Sigma_{n}\left(\left|t_{n, \uparrow}^{S}\right|^{2}-\left|t_{n, \downarrow}^{S}\right|^{2}\right)$ is the magnetoresistance term which leads to a spin polarization of the current, and $G_{\phi}=2 G_{Q} \Sigma_{n}\left(\rho_{n}^{F}-4\left[\tau_{n}^{S} / T_{n}\right]\right)$ is the phase-shifting conduc- 
tance, with $\tau_{n}^{S}=\operatorname{Im}\left[t_{n, \uparrow}^{S} t_{n, \downarrow}^{S *}\right], \rho_{n}^{F}=\operatorname{Im}\left[r_{n, \uparrow}^{F} r_{n, \downarrow}^{F}{ }^{*}\right]$ and $G_{Q}=e^{2} / h$. These three terms already appeared in Ref. 15 for studying normal electrodes in contact with $S$ and $F$ reservoirs (with no proximity effect in $F$ ). The extra terms in $G_{\xi}$ $=-G_{Q} \Sigma_{n} \tau_{n}^{S}$ and $G_{\chi}=G_{Q} \Sigma_{n} T_{n}\left(\rho_{n}^{F}+\tau_{n}^{S}\right) / 4$ occur because there are superconducting correlations at both sides of the interface. Note that $G_{\phi}, G_{\chi}$, and $G_{\xi}$ can be finite only if the phase shifts acquired by the electrons upon reflection or transmission at the interface are spin dependent. The exact values of these conductance coefficients depend on the microscopic structure of the interface. However, we can estimate their relative orders of magnitude in a rectangular potential barrier model by describing the ferromagnetism of $F$ with an exchange field $E_{e x}$ that is much smaller than the spin-averaged Fermi energy $E_{F}$ of $F$. This gives expressions of $G_{M R}, G_{\phi}$, $G_{\chi}$, and $G_{\xi}$ linear with $E_{e x} / E_{F}$. The tunnel limit can be reached by considering a strong mismatch between the Fermi wave vectors in $S$ and $F$ (case 1) or a high enough barrier (case 2). In both limits we find $\left|G_{M R}\right|,\left|G_{\chi}\right|,\left|G_{\xi}\right| \ll G_{t}$, which allows us to neglect these terms in the following. In case 1, we find $\left|G_{\phi}\right| \ll G_{t}$ whereas in case $2,\left|G_{\phi}\right|$ can be larger than $G_{t}$. Thus we will study the consequences of the spindependent BC for an arbitrary value of $\left|G_{\phi}\right| / G_{t}$. In addition, in case 1 we find $G_{\phi}<0$ but in case 2, the sign of $G_{\phi}$ depends on the details of the barrier, thus we will consider both signs for $G_{\phi}$.

In equilibrium, we can use normal and anomalous quasiclassical Matsubara Green's functions parametrized, respectively, as $\cos \left(\Lambda_{\sigma}\right)$ and $\sin \left(\Lambda_{\sigma}\right) \exp \left(i \varphi_{\sigma}\right)$ to describe the normal excitations and the condensate of pairs (see, e.g., Ref. 21). The spatial variations of the superconducting correlations in $F$ are described by the Usadel equations $\partial Q_{\sigma} / \partial x=0$ and

$$
\partial^{2} \Lambda_{\sigma} / \partial x^{2}=k_{\sigma}^{2} \operatorname{sgn}\left(\omega_{n}\right) \sin \left(\Lambda_{\sigma}\right) / \xi_{F}^{2}+Q_{\sigma}^{2} \cos \left(\Lambda_{\sigma}\right) / \sin ^{3}\left(\Lambda_{\sigma}\right),
$$

with $\xi_{F}=\left(\hbar D / E_{e x}\right)^{1 / 2}, \quad \omega_{n}=(2 n+1) \pi k_{B} T$. Here, $Q_{\sigma}$ $=\sin ^{2}\left(\Lambda_{\sigma}\right) \partial \varphi_{\sigma} / \partial x$ is the spectral current (constant with $x$ ) and $D$ the diffusion coefficient. We introduced $k_{\sigma}$ $=\left\{2\left[i \sigma \operatorname{sgn}\left(\omega_{n}\right)+\left|\omega_{n}\right| / E_{e x}\right]\right\}^{1 / 2}$ for later use. ${ }^{2}$ Neglecting $G_{M R}$, $G_{\chi}$, and $G_{\xi}$ in (1) yields

$$
\begin{gathered}
g_{F} \frac{\partial \Lambda_{\sigma}}{\partial x}=i G_{\phi} \sigma \sin \left(\Lambda_{\sigma}\right)+G_{t}\left[\cos \left(\Lambda_{S}\right) \sin \left(\Lambda_{\sigma}\right)\right. \\
\left.-\sin \left(\Lambda_{S}\right) \cos \left(\Lambda_{\sigma}\right) \cos \left(\varphi_{\sigma}-\varphi_{S}\right)\right] \\
g_{F} \frac{\partial \varphi_{\sigma}}{\partial x} \sin \left(\Lambda_{\sigma}\right)=G_{t} \sin \left(\Lambda_{S}\right) \sin \left(\varphi_{\sigma}-\varphi_{S}\right)
\end{gathered}
$$

In Eqs. (2) and (3), we used rigid BC for $S$, i.e., $\Lambda_{\sigma}=\Lambda_{S}$ $=\arctan \left[\Delta / \omega_{n}\right]$, with $\Delta$ the gap of $S$.

In the following, we consider the limit of a weak proximity effect in $F$, i.e., $\Lambda_{\sigma}=\theta_{\sigma}$ for $\omega_{n}>0$ and $\Lambda_{\sigma}=\pi-\theta_{\sigma}$ for $\omega_{n}<0$ with $\left|\theta_{\sigma}(x)\right| \ll 1$. We first study geometries with $Q_{\sigma}$ $=0$, i.e., no supercurrent flows through the device. In this case, the proximity effect in $F$ can be probed through measurements of the density of states $N(\varepsilon)=N_{0}\{1$ $\left.-\Sigma_{\sigma} \operatorname{Re}\left[\theta_{\sigma}^{2}(x)\right] / 4\right\}$ [with $\omega_{n}=-i \varepsilon+0^{+}$and $\operatorname{sgn}\left(\omega_{n}\right)=1$ ]. The simplest case of a single $S / F$ interface with $F$ at $x>0$ yields

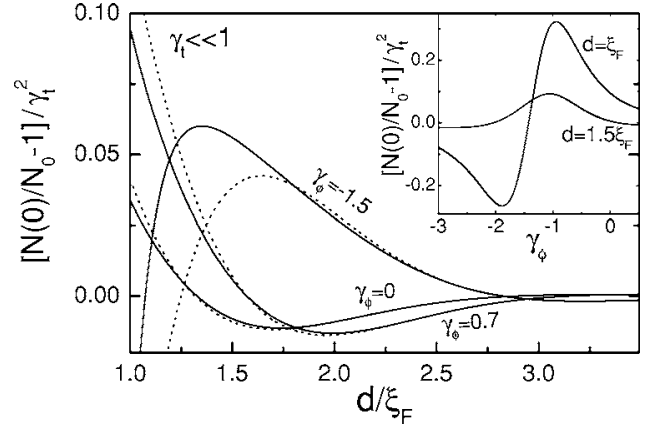

FIG. 1. Zero energy density of states at $x=d$ in a $S / F / I$ structure, in terms of $\left\{\left[N(0) / N_{0}\right]-1\right\} / \gamma_{t}^{2}$ as a function of $d / \xi_{F}$, for $\gamma_{t}$ $\ll 1$ and different values of $\gamma_{\phi}$ (full lines). The dotted lines show $4\left[\left[N(0) / N_{0}\right]-1\right] / \gamma_{t}^{2}$ at $x=d$ in a semi-infinite $S / F$ structure with the same values of $\gamma_{t}$ and $\gamma_{\phi}$. The inset shows the DOS at $x=d$ as a function of $\gamma_{\phi}$ for the $S / F / I$ structure.

$$
\theta_{\sigma}^{S F}(x)=\frac{\gamma_{t} \sin \left(\Lambda_{S}\right)}{\gamma_{t}\left|\cos \left(\Lambda_{S}\right)\right|+i \gamma_{\phi} \sigma \operatorname{sgn}\left(\omega_{n}\right)+k_{\sigma}} e^{-k_{\sigma}^{x / \xi_{F}}}
$$

with $\gamma_{t(\phi)}=G_{t(\phi)} \xi_{F} / g_{F}$. In the limit $\Delta \ll E_{e x}$ where $k_{\sigma}=1$ $+i \sigma \operatorname{sgn}\left(\omega_{n}\right)$, the weak proximity effect hypothesis leading to (4) is valid for any values of $\gamma_{\phi}$ and $\varepsilon$ if $\gamma_{t} \ll 1$. Since $k_{\sigma}$ has finite real and imaginary parts, $\theta_{\sigma}^{S F}(x)$ shows the well-known exponentially damped sinusoidal oscillations with $d$. The remarkable point in (4) is that $\gamma_{\phi}$ shifts these oscillations and modifies their amplitude [see Fig. 1 which shows the DOS following from (4)]. We also study the $S / F / I$ geometry, with $F$ at $x \in[0, d]$ and the insulating layer $I$ at $x>d$, for later comparison with the experimental data of Ref. 3. Using (2) for the $S / F$ interface and $\partial \theta_{\sigma} / \partial x=0$ for $F / I$ yields

$$
\theta_{\sigma}^{S F I}(x)=\theta_{\sigma}^{d} \cosh \left((x-d) \frac{k_{\sigma}}{\xi_{F}}\right)\left[\cosh \left(k_{\sigma} \frac{d}{\xi_{F}}\right)\right]^{-1}
$$

with

$$
\theta_{\sigma}^{d}=\frac{\gamma_{t} \sin \left(\Lambda_{S}\right)}{\left[\gamma_{t}\left|\cos \left(\Lambda_{S}\right)\right|+i \gamma_{\phi} \sigma \operatorname{sgn}\left(\omega_{n}\right)+k_{\sigma} \tanh \left(k_{\sigma} d / \xi_{F}\right)\right]} .
$$

In the limit $\Delta \ll E_{e x}$ and $d \geqslant \xi_{F}$, the $\theta$ linearization leading to (5) is again valid for any $\gamma_{\phi}$ and $\varepsilon$ if $\gamma_{t} \ll 1$. From Fig. $1, \gamma_{\phi}$ has qualitatively the same effect on $\theta_{\sigma}^{S F I}(x)$ as on $\theta_{\sigma}^{S F}(x)$. More quantitatively, for $d \gg \xi_{F}$ one has $\theta_{\sigma}^{S F I}(x=d) / \theta_{\sigma}^{S F}(x$ $=d)=2$ (Ref. 22) and for lower values of $d$, this ratio depends on $d$.

Another way to probe the superconducting proximity effect in $F$ is to measure the supercurrent through a $S / F / S$ Josephson junction. We consider a junction with $F$ at $x$ $\in[0, d]$ and a right (left) superconducting reservoir, called $R(L)$ at a constant phase $(-) \varphi_{S} / 2$. A supercurrent $I_{S}$ $=\pi g_{F} k_{B} T \Sigma_{n \in Z, \sigma= \pm 1} Q_{\sigma}\left(\omega_{n}\right) / 2 e$ flows through this device. ${ }^{2} \mathrm{We}$ focus on the asymmetric limit $\gamma_{t}^{R} \ll \gamma_{t}^{L}$, which corresponds to the experiment of Ref. 5, and assume $\gamma_{\phi}^{R}=0 .{ }^{23}$ We allow $L$ and $R$ to have different superconducting gaps $\Delta^{R(L)}$, so that $\Lambda_{\sigma}=\Lambda_{S}^{R(L)}$ in $R(L)$. Solving this problem perturbatively with respect to the $S / F / I$ case yields 


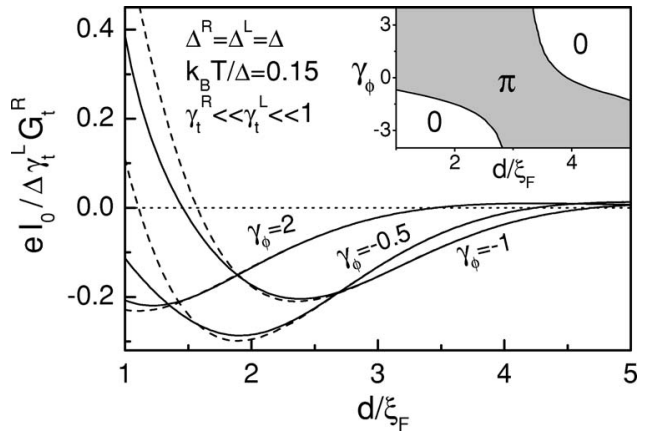

FIG. 2. Critical current $I_{0}$ of an asymetric $S / F / S$ junction as a function of $d / \xi_{F}$, calculated from Eq. (6) for $\gamma_{t}^{R} \ll \gamma_{t}^{L} \ll 1, \Delta^{L(R)}$ $=\Delta \ll E_{e x}$ and $k_{B} T / \Delta=0.15$ (full lines). The dashed lines show the large $d / \xi_{F}$ approximation of Eq. (7). The inset is a phase diagram indicating the equilibrium state of the junction $(0$ or $\pi)$ depending on $\gamma_{\phi}$ and $d / \xi_{F}$.

$$
Q_{\sigma}\left(\omega_{n}\right)=\theta_{\sigma}^{d} \gamma_{t}^{R} \sin \left(\Lambda_{S}^{R}\right) \sin \left(\varphi_{S}\right)\left[\xi_{F} \cosh \left(k_{\sigma} \frac{d}{\xi_{F}}\right)\right]^{-1},
$$

where $\theta_{\sigma}^{d}$ corresponds to the expression given above with $\Lambda_{S}=\Lambda_{S}^{L}$ and $\gamma_{t(\phi)}=\gamma_{t(\phi)}^{L}$. The supercurrent has the form $I_{S}$ $=I_{0} \sin \left(\varphi_{S}\right)$ because most of the phase drop occurs at $R$. In the limit $\Delta^{L}=\Delta^{R}=\Delta \ll E_{e x}, \gamma_{t}^{L} \ll 1$ and $d / \xi_{F} \gg 1$, (6) yields

$$
\frac{e I_{0}}{\gamma_{t}^{L} G_{t}^{R} \Delta}=\pi \tanh \left(\frac{\Delta}{2 k_{B} T}\right)\left[\frac{\sin \left(\frac{d}{\xi_{F}}+\lambda\left(\gamma_{\phi}^{L}\right)\right)}{\left[1+\left(1+\gamma_{\phi}^{L}\right)^{2}\right]^{1 / 2}} e^{-d / \xi_{F}}\right]
$$

with $\lambda\left(\gamma_{\phi}^{L}\right)=\arg \left[i-\left(1+\gamma_{\phi}^{L}\right)\right]$. It is already known that the state of the junction depends on $d$. Equation (7) shows that $\gamma_{\phi}^{L}$ shifts the oscillations of the $I_{0}(d)$ curve. Thus, for a given value of $d$, the state of the junction can be 0 as well as $\pi$, depending on $\gamma_{\phi}^{L}$. Figure 2 shows that this effect still occurs when one goes beyond the large $d / \xi_{F}$ approximation. Note that in the limit $\Delta \ll E_{e x}$ and $\gamma_{t}^{L} \ll 1$ used to obtain (7), it is not possible to find a temperature crossover for the sign of $I_{0}$ as observed in Refs. 4 and 6. However, we expect to find such a temperature crossover with a $0 / \pi$ or $\pi / 0$ transition, depending on the value of $\gamma_{\phi}^{L}$, if the energy dependence of $k_{\sigma}$ is taken into account. ${ }^{25}$

To show the relevance of our approach, we compare our predictions with the measurements of Refs. 3 and 5. We first consider the $\left|I_{0}\right|$ measured in an asymmetric $S / F / S$ junction, i.e., $\mathrm{Nb} / \mathrm{Pd}_{1-x} \mathrm{Ni}_{x} / \mathrm{Alox} / \mathrm{Al} / \mathrm{Nb}$ with $x \sim 0.1$ and $\gamma_{t}^{L} / \gamma_{t}^{R}$ $\sim 10^{5}$. We assume that the contacts have $T_{n} \ll 1$, which allows to use Eqs. (2) and (3). We will use the experimentally determined values $\Delta^{A l / N b}=0.6 \mathrm{meV}$ and $\Delta^{N b}=1.35 \mathrm{meV}$ $\ll E_{\text {ex }}$, which implies $k_{\sigma} \sim 1+i \sigma$, and $T=1.5 \mathrm{~K}$. Samples with different thicknesses $d$ of PdNi were measured (see Fig. 3). Interpreting these data requires a careful analysis of the influence of $d$ on the different parameters. We have $g_{F}$ $=2 e^{2} N_{0} D A$ and $\xi_{F}=\sqrt{\hbar D / E_{e x}}$, with $D=v_{F} l / 3$ and $A$ the conductors cross section. Curie temperature measurements show that the exchange field $E_{e x}$ increases linearly with $d .^{26}$ In addition, we first assume that the mean free path $l$ is constant with $d$, as confirmed by resistivity measurements for $d>d_{0}$

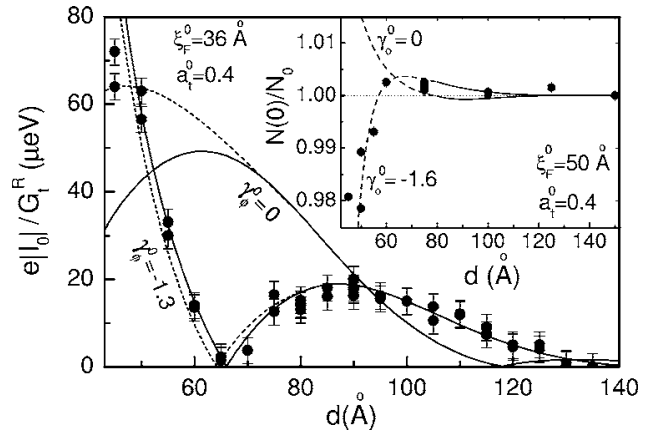

FIG. 3. Critical current measured as in Ref. 5 through $\mathrm{Nb} / \mathrm{Pd}_{1-x} \mathrm{Ni}_{x} / \mathrm{Alox} / \mathrm{Al} / \mathrm{Nb}$ junctions as a function of the thickness $d$ of $\mathrm{Pd}_{1-x} \mathrm{Ni}_{x}$ (symbols). The lines are theoretical curves calculated from Eq. (6) for $d>d_{0}$ (full lines) and $d<d_{0}$ (dashed lines) with the fitting parameters $a_{t}^{0}=0.4, \xi_{F}^{0}=36 \AA$ and the experimentally determined parameters $\Delta^{N b}=1.35 \mathrm{meV}, \Delta^{A l / N b}=0.6 \mathrm{meV}, d_{0}=80 \AA$ and $T=1.5 \mathrm{~K}$. The data are well fitted with $\gamma_{\phi}^{0}=-1.3$. We also show the theory for $\gamma_{\phi}^{0}=0$. Inset: DOS measured by Ref. 3 in $\mathrm{Nb} /$ $\mathrm{Pd}_{1-x} \mathrm{Ni}_{x} / \mathrm{Alox} / \mathrm{Al}$, as a function of $d$. The full and dotted lines show the DOS at $x=d$ calculated from the second-order generalization of (5) (see text), for $d>d_{0}$ and $d<d_{0}$, respectively. We used $\xi_{F}^{0}=50 \AA$ and $\gamma_{\phi}^{0}=-1.6$ or $\gamma_{\phi}^{0}=0$, all the other parameters being unchanged.

$=80 \AA$. This allows us to parametrize the problem with $\gamma_{t}^{L}$ $=a_{t}^{0} \xi_{F}^{0} / \sqrt{d_{0} d}$ and $\xi_{F}=\xi_{F}^{0} \sqrt{d_{0} / d}$ where $\xi_{F}^{0}$ and $a_{t}^{0}=G_{t}^{L} d_{0} / g_{F}$ are constant with $d$. We also assume that $G_{\phi}^{L}$ is proportional to $E_{e x}$ as found above in the rectangular barrier model for $E_{e x}$ $\ll E_{F}$, so that we take $\gamma_{\phi}^{L}=\gamma_{\phi}^{0} \sqrt{d / d_{0}}$ with $\gamma_{\phi}^{0}$ constant with $d$. We neglect $\gamma_{\phi}^{R}$ due to the existence of a strong insulating barrier at $R .^{24}$ The absolute amplitude of $E_{e x}$ was not determined exactly, so that $\xi_{F}^{0}$ can be considered as a fitting parameter as well as $a_{t}^{0}$ and $\gamma_{\phi}^{0}$. This makes in total three fitting parameters but we expect to find for $a_{t}^{0}$ a value close to the value 0.2 found from minigap measurements in $\mathrm{Nb} / \mathrm{Pd} .{ }^{25} \mathrm{We}$ have calculated $\left|I_{0}\right|$ by summing (6) on energy and spin. It is not possible to account for the data with $\gamma_{\phi}^{0}=0$. On the contrary, a good agreement with the experiment is obtained by using $a_{t}^{0}=0.4, \xi_{F}^{0}=36 \AA$, and $\gamma_{\phi}^{0}=-1.3$ (full lines in Fig. $3) .26,27$ We have checked that this choice of parameters fulfills the hypothesis $\left|\theta_{\sigma}(x)\right| \ll 1$ made in our calculations. Remarkably, for $d \sim d_{0}$ in Fig. 3, the theory for $\gamma_{\phi}^{0}=-1.3$ gives $I_{0}<0$ in agreement with subsequent experiments, ${ }^{27,28}$ whereas it gives $I_{0}>0$ for $\gamma_{\phi}^{0}=0$ if one keeps the same orders of magnitude for $a_{t}^{0}$ and $\xi_{F}^{0}$. For $d<d_{0}, l$ is linear with $d$, which we have taken into account by using $\xi_{F}=\xi_{F}^{0}, \gamma_{\phi}^{L}=\gamma_{\phi}^{0}$, and $\gamma_{t}^{L}=a_{t}^{0} \xi_{F}^{0} / d$, with the same values of $a_{t}^{0}, \gamma_{\phi}^{0}$, and $\xi_{F}^{0}$ as previously given (dashed lines in Fig. 3). This approach gives a surprising agreement with the data, which seems to indicate that the Usadel description still works for $d<d_{0}$ although $l$ is linear with $d .{ }^{29}$ Kontos et al. have also performed DOS measurements in $\mathrm{Nb} / \mathrm{Pd}_{1-x} \mathrm{Ni}_{x} / \mathrm{Alox} / \mathrm{Al},{ }^{3}$ prior to the $I_{0}$ measurements. We have assumed again that $E_{e x}$ was linear with $d$ in these measurements, to try to interpret the $N(0)=f(d)$ curve with the same fitting procedure as for $I_{0}$. We have generalized Eq. (5) to second order in $\theta_{\sigma}$ because the values of $d / \xi_{F}$ are slightly lower than for the $I_{0}$ measure- 
ments. Again it is impossible to interpret the data with $\gamma_{\phi}^{0}$ $=0$. We obtain a satisfactory fit by choosing $\xi_{F}^{0}=50 \AA$ and $\gamma_{\phi}^{0}=-1.6$, all the other parameters used being the same as in the previous case. Finding a $\xi_{F}^{0}$ higher than for the $I_{0}$ data is in agreement with the fact that the samples used for measuring the DOS were realized with a lower concentration $x$ of Ni.

In summary, we have studied the effect of spin-dependent boundary conditions on the superconducting proximity effect in a diffusive ferromagnet bounded by tunneling interfaces. We have shown that the phase-shifting conductances $G_{\phi}$, describing the spin activity of the interfaces in this context, strongly affect the behavior of the system and allow a consistent microscopic explanation of the DOS and supercurrent data of Refs. 3 and 5. This suggests that such effects will have to be considered in any future work on $S / F$ hybrid circuits. In the context of spintronics, this approach might also provide a way to characterize spin-active interfaces.

We thank T. Kontos for raising the question which led us to perform this study and for providing us with the experimental data. We thank C. Bruder, T. T. Heikkilä, and D. Huertas-Hernando for discussions. This work was financed by the Swiss NSF and the NCCR Nanoscience.
${ }^{1}$ A. I. Buzdin, L. N. Bulaevskii, and S. V. Panjukov, JETP Lett. 35, 178 (1982).

${ }^{2}$ A. A. Golubov, M. Yu. Kupriyanov, and E. Il'ichev, Rev. Mod. Phys. 76, 411 (2004).

${ }^{3}$ T. Kontos, M. Aprili, J. Lesueur, and X. Grison, Phys. Rev. Lett. 86, 304 (2001).

${ }^{4}$ V. V. Ryazanov, V. A. Oboznov, A. Yu. Rusanov, A. V. Veretennikov, A. A. Golubov, and J. Aarts, Phys. Rev. Lett. 86, 2427 (2001).

${ }^{5}$ T. Kontos, M. Aprili, J. Lesueur, F. Genêt, B. Stephanidis, and R. Boursier, Phys. Rev. Lett. 89, 137007 (2002).

${ }^{6}$ H. Sellier, C. Baraduc, F. Lefloch, and R. Calemczuk, Phys. Rev. B 68, 054531 (2003).

${ }^{7}$ Y. Blum, A. Tsukernik, M. Karpovski, and A. Palevski, Phys. Rev. Lett. 89, 187004 (2002).

${ }^{8}$ W. Guichard, M. Aprili, O. Bourgeois, T. Kontos, J. Lesueur, and P. Gandit, Phys. Rev. Lett. 90, 167001 (2003).

${ }^{9}$ L. B. Ioffe, V. B. Geshkenbein, M. V. Feigel'man, A. L. Fauchère, G. Blatter, Nature (London) 398, 679 (1999).

${ }^{10}$ D. H. Hernando, Yu. V. Nazarov, A. Brataas, and G. E. W. Bauer, Phys. Rev. B 62, 5700 (2000); A. Brataas, Y. V. Nazarov, and G. E. W. Bauer, Eur. Phys. J. B 22, 99 (2001); A. Brataas, Yu. V. Nazarov, and G. E. W. Bauer, Phys. Rev. Lett. 11, 2481 (2000).

${ }^{11}$ W. Wetzels, G. E. W. Bauer, and M. Grifoni, Phys. Rev. B 72, 020407(R) (2005).

${ }^{12}$ L. Balents and R. Egger, Phys. Rev. B 64, 035310 (2001).

${ }^{13}$ T. Tokuyasu, J. A. Sauls, and D. Rainer, Phys. Rev. B 38, 8823 (1988)

${ }^{14}$ A. Millis, D. Rainer, and J. A. Sauls, Phys. Rev. B 38, 4504 (1988); M. Fogelström, ibid. 62, 11812 (2000); J. C. Cuevas and M. Fogelström, ibid. 64, 104502 (2001); N. M. Chtchelkatchev, W. Belzig, Yu. V. Nazarov, and C. Bruder, JETP Lett. 74, 323 (2001); J. Kopu, M. Eschrig, J. C. Cuevas, and M. Fogelström, Phys. Rev. B 69, 094501 (2004); E. Zhao, T. Löfwander, and J. A. Sauls, ibid. 70, 134510 (2004).
${ }^{15}$ D. H. Hernando, Yu. V. Nazarov, and W. Belzig, Phys. Rev. Lett. 88, 047003 (2002); cond-mat/0204116 (unpublished).

${ }^{16}$ P. M. Tedrow, J. E. Tkaczyk, and A. Kumar, Phys. Rev. Lett. 56, 1746 (1986).

${ }^{17}$ M. Yu. Kuprianov and V. F. Lukichev, JETP Lett. 67, 1163 (1988).

${ }^{18}$ J. M. E. Geers, M. B. S. Hesselberth, J. Aarts, and A. A. Golubov, Phys. Rev. B 64, 094506 (2001).

${ }^{19}$ T. Kontos, M. Aprili, J. Lesueur, X. Grison, and L. Dumoulin, Phys. Rev. Lett. 93, 137001 (2004).

${ }^{20}$ Yu. V. Nazarov, Phys. Rev. Lett. 73, 1420 (1994); Superlattices Microstruct. 25, 1221 (1999).

${ }^{21}$ W. Belzig, F. K. Wilhelm, C. Bruder, G. Schön, and A. D. Zaikin, Superlattices Microstruct. 25, 1251 (1999).

${ }^{22}$ This follows from superposing two semi-infinite solutions like Eq. (4), which is possible for $d \gg \xi_{F}$ [see A. I. Buzdin, B. Bujicic, and M. Yu. Kupriyanov, Sov. Phys. JETP 74, 124 (1992)].

${ }^{23}$ With a high (rectangular) barrier, one has $G_{t} \ll\left|G_{\phi}\right|$, but if the barrier is too high, one can check that $I_{0}$ is unaffected by $G_{\phi}^{R}$ because $G_{\phi}^{R} \ll G_{t}^{L}, G_{\phi}^{L}$.

${ }^{24}$ This will be investigated elsewhere.

${ }^{25}$ T. Kontos, Ph.D. thesis, Université Paris-Sud, Orsay, France, 2002.

${ }^{26}$ Another interpretation of Ref. 5 was proposed in Ref. 27 for $\gamma_{\phi}$ $=0$, but with values of $\Delta_{L} \Delta_{R}$ and $T$ in disagreement with the experimentally determined parameters and continuous BC $\Lambda_{\sigma}$ $=\Lambda_{S}^{L}$ for $x=0$.

${ }^{27}$ A. Buzdin and I. Baladié, Phys. Rev. B 67, 184519 (2003).

${ }^{28}$ A. Bauer, J. Bentner, M. Aprili, M. L. Della-Rocca, M. Reinwald, W. Wegscheider, and C. Strunk, Phys. Rev. Lett. 92, 217001 (2004).

${ }^{29}$ The ballistic approach of M. Zareyan, W. Belzig, and Yu. V. Nazarov, Phys. Rev. Lett. 86, 308 (2001); Phys. Rev. B 65, 184505 (2002) could not account for the full $d$ dependence of Ref. 5. 